\title{
Biological Fingerprinting of Herbal Samples by Means of Liquid Chromatography
}

\author{
Lukasz Cieśla ${ }^{1,2}$ \\ ${ }^{1}$ Department of Inorganic Chemistry, Medical University of Lublin, Chodźki 4a, 20-093 Lublin, Poland \\ ${ }^{2}$ Department of Biochemistry, Institute of Soil Science and Plant Cultivation, State Research Institute, \\ Czartoryskich 8, 24-100 Puławy, Poland
}

Correspondence should be addressed to Łukasz Cieśla, lukecarpenter@poczta.onet.pl

Received 15 July 2011; Accepted 22 August 2011

Academic Editor: Teresa Kowalska

Copyright ( 2012 Łukasz Cieśla. This is an open access article distributed under the Creative Commons Attribution License, which permits unrestricted use, distribution, and reproduction in any medium, provided the original work is properly cited.

\begin{abstract}
Biological chromatographic fingerprinting is a relatively new concept in the quality control of herbal samples. Originally it has been developed with the application of HPLC, and recently herbal samples' biological profiles have been obtained by means of thin-layer chromatography (TLC). This paper summarizes the application of liquid chromatographic techniques for the purpose of biological fingerprint analysis (BFA) of complex herbal samples. In case of biological TLC fingerprint, which is a relatively novel solution, perspectives of its further development are outlined in more detail. Apart from already published data, some novel results are also shown and briefly discussed. The paper aims at drawing scientists' attention to the unique solutions offered by biological fingerprint construction.
\end{abstract}

\section{Introduction}

Fingerprint construction has become an important quality control tool of herbal samples in the light of constantly growing interest in natural origin medicines. Fingerprint analysis has been accepted by WHO as a methodology for the quality control of herbal samples $[1,2]$. It is applied to identify closely related plant species, to detect adulterations, to control the extraction process or to study the quality of a finished product. Herbal sample fingerprint can be defined as a set of characteristic chromatographic or spectroscopic signals, whose comparison leads to an unambiguous sample recognition. Several chromatographic methods have been applied for fingerprint construction, namely, high-performance liquid chromatography (HPLC), thinlayer chromatography (TLC), gas chromatography (GC), or high-speed counter current chromatography (HSCCC). However, it is difficult to indicate with $100 \%$ certainty which signals (peaks, bands, etc.) should be present in the obtained fingerprint to confirm sample identity. For that purpose the analyzed sample can be compared with a defined Botanical Reference Material (BRM) or a set of standard compounds. Defining and obtaining BRM for every plant species is a difficult task, therefore, new solutions are sought for fingerprint comparison. More recently a concept of multiple fingerprints construction and multidimensional fingerprinting have gained much attention, as large amount of chromatographic and/or spectroscopic signals enable more comprehensive data analysis [3]. Multiple chromatographic fingerprint consists of more than one chromatographic profile [3], while in multidimensional fingerprinting hyphenated detectors are used (e.g., DAD and MS) that both record eluting compounds [1]. Usually the set of obtained data is further processed by means of similarity measure or chemometrics approach (e.g., principal component analysis, hierarchical clustering, multivariate calibration, etc.) [1,3]. In case of multiple fingerprints, a data fusion-based method is proposed [3]. It is also a common approach to construct the reference/standard fingerprints that are further used for data comparison. The similarity of a tested sample's fingerprint and a reference chromatographic profile is performed usually using the correlation coefficient measure [3].

As far as herbal medicines are considered their biological activity is an important issue. However, traditional chromatographic fingerprint analysis provides the researchers 
only with qualitative and quantitative information. A critical issue is the fact, that compounds present in low concentration may exert more potent biological activity than those present in greater amounts. It is, therefore, important to introduce screening of the biological activity into the chromatographic fingerprint analysis. To screen the natural samples for the presence of most active compounds biological fingerprinting analysis has been introduced. It was originally developed with the use of high-performance liquid chromatography. Apart from qualitative and quantitative data, it gives also the possibility to fish out the active compounds from a myriad of compounds present in herbal samples. HPLC biological fingerprinting techniques have been well reviewed by Su et al. [4] and Yu et al. [5].

\section{Biological Fingerprinting by Means of HPLC}

The concept of biological fingerprinting has been first developed for the purposes of the quality control of complex traditional Chinese medicines [4]. By analogy with the definition of traditional chromatographic fingerprint, biological fingerprint (biofingerprint) can be defined as a set of chromatographic and/or spectroscopic signals that enable the identification of the active compounds present in a complex herbal sample. Biological fingerprints have been also coupled with traditional chromatographic profiles (binary fingerprints) for obtaining more comprehensive information on the complex sample. However, the main goal of biofingerprint construction is to pinpoint individual active compounds present in a complex matrix, that can, for example, constitute good candidates for potential drugs. Biofingerprint analysis can also be used to simulate and evaluate the actions of active compounds in vivo (e.g., interaction with cell membranes, receptors, enzymes, serum proteins, etc.) [6]. Therefore, biofingerprints combine the data obtained in traditional chromatographic fingerprint (qualitative and quantitative) with biological activity. The majority of biofingerprints have been obtained basing on small molecule-biomacromolecule interactions [4]. The compounds present in herbal formulations were checked for their possible interactions with DNA, serum proteins, liver homogenate, or liposomes [4]. Usually RP-HPLC column has been coupled with a column containing immobilized proteins or other macromolecules (affinity chromatography). The most popular techniques have been those using DNA, as it is a molecular target of many drugs, for example, anticancer, antiviral, or antibacterial [4]. To construct biological profiles, two different approaches have been used: affinity chromatography with immobilization of DNA onto silica gel [7] and microdialysis after the interaction with DNA [8]. In the former case, the authors used immobilized DNA column with silica monolithic ODS column. With the application of such chromatographic conditions, the authors constructed binary fingerprints for Coptis chinensis Franch and Rheum palmatum (see Figure 1). The major drawback of such solution is the column efficiency decrease due to DNA degradation; therefore, the column should be stored at $4^{\circ} \mathrm{C}$ [7]. In case of the method with microdialysis step,

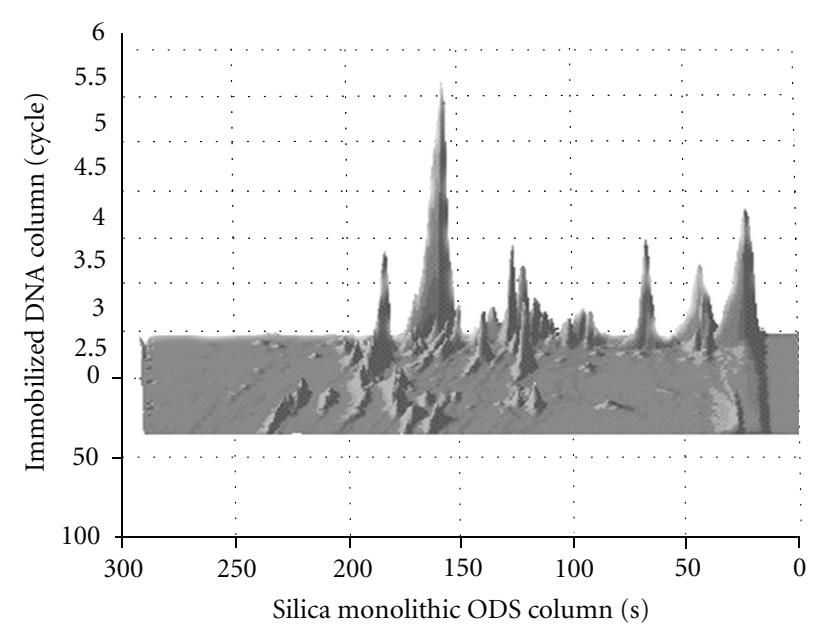

FIGURE 1: Binary chromatographic fingerprint obtained for Rheum palmatum L. For experimental details, see publication by $\mathrm{Su}$ et al. [7] (originally published in [7]).

the authors compared chromatographic profiles obtained before and after the interaction with DNA [8]. Apart from data multiplication, which eases the comparative studies, DNA-binding fingerprint gives an opportunity to indicate compounds with a potential to be used as DNA-target drugs. Biofingerprinting chromatogram analysis constitutes also an alternative for a conventional procedure for discovery of bioactive compounds present in complex samples.

Apart from DNA-interaction profiles, biological fingerprints have been developed with the use of human serum albumin (HSA) immobilized on a surface of a column packing [9]. This protein is one of the most important drug-binding macromolecules in human plasma. Wang et al. applied silica-bonded human serum albumin column in the first direction and monolithic ODS column in the second one to screen traditional Chinese medicine prescription consisting of ten medicinal materials [9]. The authors underline the importance of applying HSA-immobilized column in the first dimension, because ODS as second-dimensional column is characterized with greater peak capacity and it is better suited for MS detection [9]. With the application of this multidimensional liquid chromatography system, 100 compounds interacting with HSA were separated and 19 of them identified (see Figure 2). The use of monolithic column, characterized with high permeability and excellent mass-transfer properties, enabled fast separation. The authors underline the need for coupling biochromatography fingerprints with traditional chromatographic profiles (2D system), as in the case of the former ones, low column efficiency and peak capacity limit its application as single fingerprint technique. 2D biochromatography was found to be advantageous for biological fingerprint analysis of complex samples, for example, traditional Chinese medicines [9].

The microdialysis step coupled with HPLC has been also used to screen the interaction of herbal samples' components with human plasma proteins [10] and cells [6]. One of the 


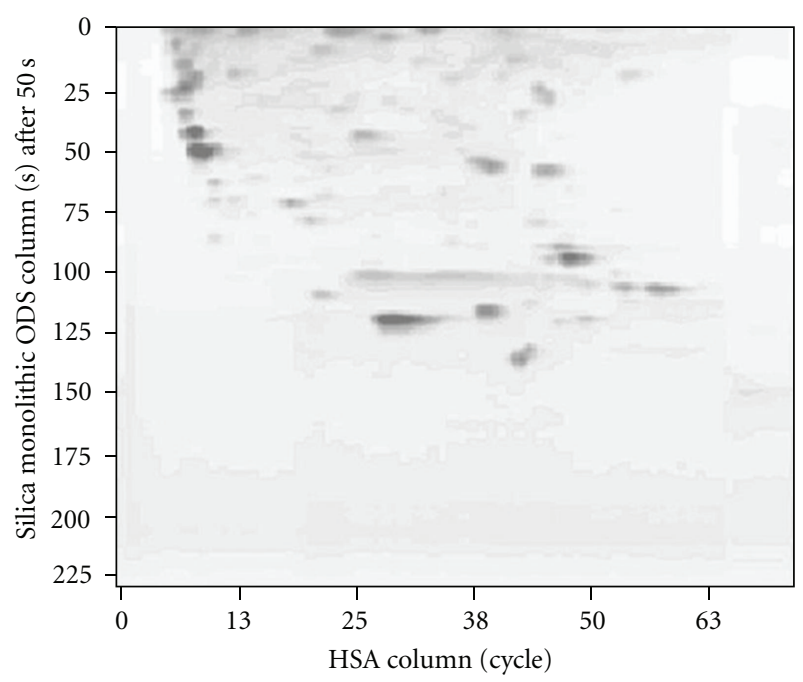

FIGURE 2: Binary chromatographic fingerprint obtained for Longdan Xiegan Decoction (originally published in [9]).

advantages of the proposed solutions is the possibility of direct sample's injection into the HPLC system after the microdialysis [10]. In case of analyte-protein interactions, the authors studied the binding degree of Rhizoma Chuanxiong components to human serum albumin (HSA) and other human blood serum proteins [10]. It was shown that the binding degree of the analytes was influenced by $\mathrm{pH}$, what has been explained as the result of the changing ionization degree of the active herbal compounds and changes in the structure of the HSA binding site. The importance of biological fingerprinting based on analyte-blood protein interactions is also explained, as molecules with lower and higher binding degrees would not be probably active after oral administration [10]. In case of the other paper, mentioned in this paragraph, biological fingerprinting analysis was used to reveal the character of anticancer activities of bioactive compounds in Cortex Pseudolarix and Radix Stephaniae [6]. The compounds that interacted with drug sensitive human breast cancer MCF-7 cells and multidrug-resistant MCF7/ADR cells were confined in the semipermeable membrane. The peaks' areas of bioactive compounds decreased when compared with chromatographic fingerprints obtained for the non-microdialised samples. The authors report on the possibility of obtaining false-positive results; however, a solution for excluding such results is also proposed [6]. It is also indicated that low recoveries of some compounds can be a limitation of microdialysis that is performed prior to HPLC analysis. The construction of biological fingerprints, basing on analyte-cancer cells interactions, can be a valuable tool to identify the potential anticancer drugs in complex natural samples [6].

Biological profile of a herbal sample can also be obtained with the application of immobilized liposome chromatography (ILC), as originally reported by Mao et al. [11]. ILC can also be a valuable tool to study drug-membrane interactions. With the application of this technique, it is possible to identify compounds that can penetrate through biological membranes, as immobilized liposomes resemble the bilayer structure of phospholipid cellular membrane. Using this technique, the authors have tested Angelica sinensis sample to evaluate the permeability of its compounds. Due to a complexity of drug-biological membrane interactions in ILC (combination of hydrophobic, ion pairing and hydrogen bonding) it is assumed to be a better model for compounds' permeability testing when compared with models based on interactions with ODS column surface [11]. Limitations concerning the use of some organic solvents and mobile phase additives, which may cause liposome bilayer destruction, are the major drawbacks of ILC. As the ILC could not be directly coupled with MS, a complementary RP-HPLC method was developed to identify the permeable compounds. Coupling ILC column with RP column has also been reported for the screening of a complex traditional Chinese medicine, Longdan Xiegan Decoction (see Figure 3) [12]. The authors identified eight flavonoids and two iridoids that could penetrate biomembranes, basing on their interactions with ILC column. As concluded by the authors, this two-dimensional system shows high suitability for biological fingerprinting analysis of complex natural samples. Reports on the use of cell membranes immobilized on chromatographic columns' packing and on their application for biological fingerprinting have also been published [4].

An interesting approach for biological fingerprint analysis has been proposed by Zhang et al., who reported on the coupling of chromatographic and metabolic fingerprint for the quality control of popular traditional Chinese prescription [13]. The chromatographic profile was obtained with the application of HPLC-UV equipment. The metabolic HPLC fingerprint was obtained after intravenous injection of the analyzed formulation in rats. After proper preparation steps, plasma samples were analyzed by means of HPLC-UV and HPLC-MS. The authors concluded that combination of chemical and metabolic fingerprints is a useful tool for the quality control and revealing possible mechanism of action of herbal samples [13]. The idea of metabolic fingerprint can be based on the concept that herbal-active compounds should be present in blood (but also in urine) after its administration [8].

Another group of biofingerprinting techniques are focused on combining traditional chromatographic fingerprint with an antioxidant profile of a sample. In this case, the sample undergoes separation only on one column; however, the separated compounds are derivatized postcolumn to check their possible antioxidant potential [14]. There are three main categories of antioxidant activity assays, namely, (1) tests that use true reactive oxygen species, (2) assays involving a relatively stable single oxidizing regents, and (3) methods applying electrochemical detection. Coupling high resolution screening techniques (HRS) with (bio)chemical detection has been reviewed by Niederländer et al. [14]. Antioxidant biofingerprinting interactions between compounds and applied derivatizing agents are of chemical rather than biological nature. Therefore, a question arises whether such fingerprints should also be termed "biological". Due to the fact that antioxidant mechanism of natural compounds in vivo seems to be analogical with that observed 


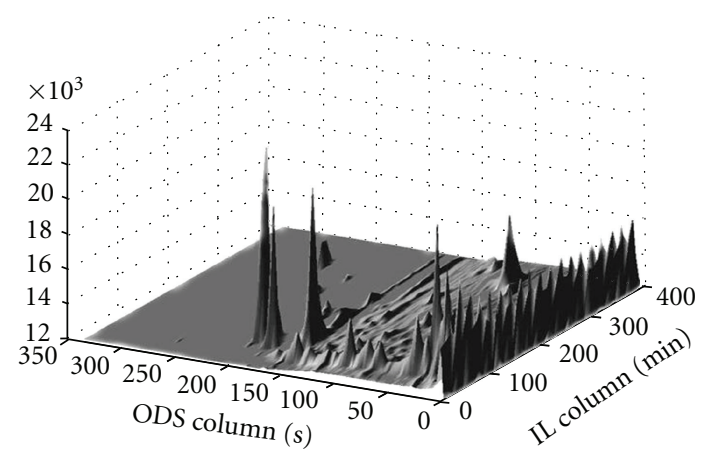

Figure 3: 3D chromatogram for Longdan Xiegan Decoction obtained by coupling immobilized liposome chromatography column in the first direction and ODS column in the second one (originally published in [12]).

in vitro (e.g., electron or hydrogen transfer to a radical [15]), these methods have also been classified, for the needs of this paper, as belonging to biological fingerprints.

Chang et al. reported on the coupling high-performance liquid chromatography with chemiluminescence detection for antioxidant activity fingerprint construction of Danshen injections (see Figure 4) [16]. Phenolic compounds able to scavenge hydrogen peroxide produced negative peaks in antioxidant-activity fingerprint.

Antioxidant activity fingerprint has been proposed for this preparation, as its protective effect on reperfusion injuries has been linked with antioxidant properties. A data fusion-based method, which combined information encoded both in antioxidant and chemical fingerprints, was applied to evaluate the investigated samples. Significant difference was observed between chromatographic profiles and activity fingerprints. Such results indicate the need to revise the common belief, that samples with similar chromatographic (chemical) profiles likely have similar properties. The authors concluded that quality control of complex herbal samples, by means of simultaneous construction of chemical and biological fingerprints, is more comprehensive when compared with traditional approach. A predominance of antioxidant-activity-integrated fingerprint over traditional chromatographic profiles has been proved for the quality control of Danshen samples.

HPLC online assays for antioxidants have been often performed with the use of postcolumn derivatization with relatively stable free radicals $\mathrm{DPPH}^{\bullet}$ or $\mathrm{ABTS}^{\bullet+}$. With the use of this technique, two profiles are always obtained: chemical (termed also "normal") with positive peaks and antioxidant profile with "negative" peaks [17]. Several solutions for the screening of complex natural samples have been proposed. They have been recently reviewed by Niederländer et al. [14] and van Beek et al. [17].

Antioxidant activity of a sample can also be predicted from its chromatographic fingerprint with the use of multivariate calibration techniques, what has been proved in a series of papers [18-23]. The authors have shown that combining the information from chromatographic fingerprints

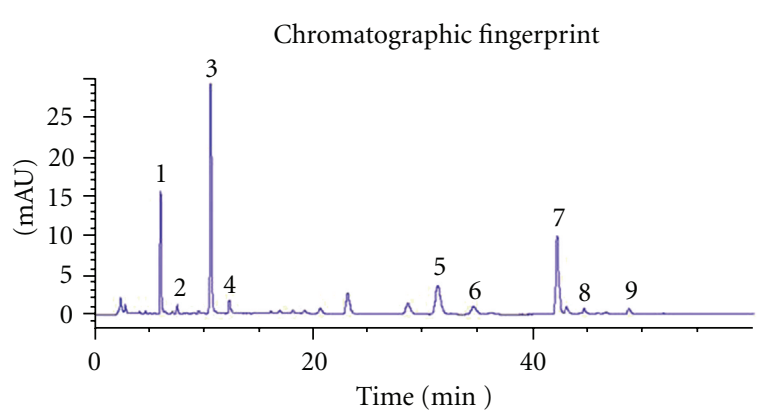

(a)

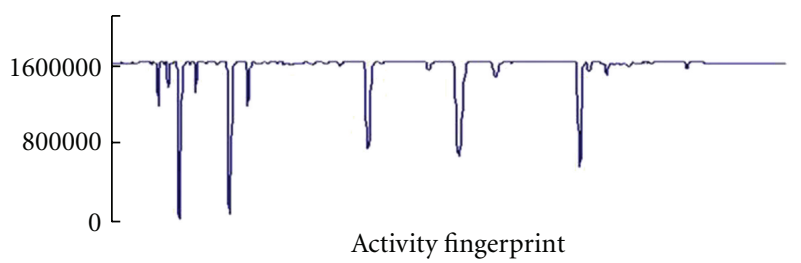

(b)

FIGURE 4: Comparison of chromatographic and antioxidant activity fingerprints of Danshen injection (originally published in [16]).

with the results obtained in spectrophotometric antioxidant assay allows good quality control of herbal samples.

There have been also trials to introduce methods aimed at identification of inhibitors of selected enzymes by means of RP-HPLC. However, their major drawbacks include: relatively large amount of enzymes needed, long reaction time as well as the unsuitability of the majority of organic solvents, used as mobile phase components, for studying analyte-enzyme interactions [17]. Further optimization steps are required to adjust these methods for routine laboratory use.

\section{Biological Fingerprinting by Means of TLC}

Thin-layer chromatography is considered to be an ideal method for fingerprint construction of herbal samples [24]. The advantages of this technique are well known and have been characterized in numerous publications. However, only a few research groups, working in the field of phytochemical analysis, are aware of its potential for biological detection. The concept of biological fingerprint development in TLC has been introduced by Cieśla et al. [25], who constructed a so-called "binary chromatographic fingerprint" combining chemical and biological detection systems. In the former case, the plates were sprayed with the use of vanillin reagent, while in the case of biological fingerprint methanolic solution of a stable free radical, $\mathrm{DPPH}^{\bullet}$, was applied (see Figure 5). Apart from videsocans, documented for fingerprint comparison, real chromatograms were obtained by means of freely available image processing programImageJ. The application of this software gives the possibility to process the fingerprints, documented in the form of videoscans without the need to use densitometers. In fact, the application of densitometers is a difficult task in case of 

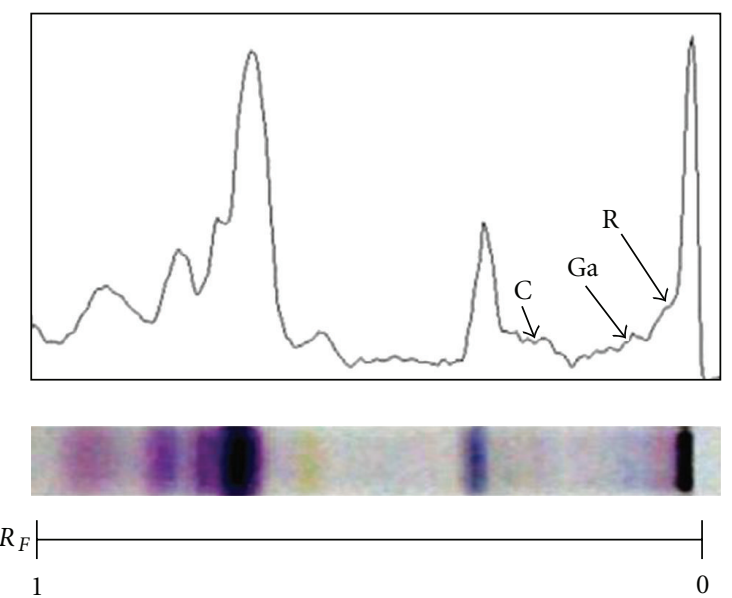

(a)
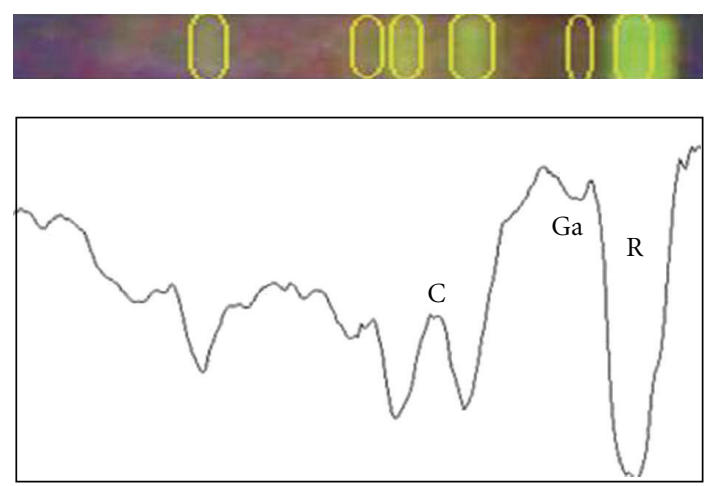

(c)
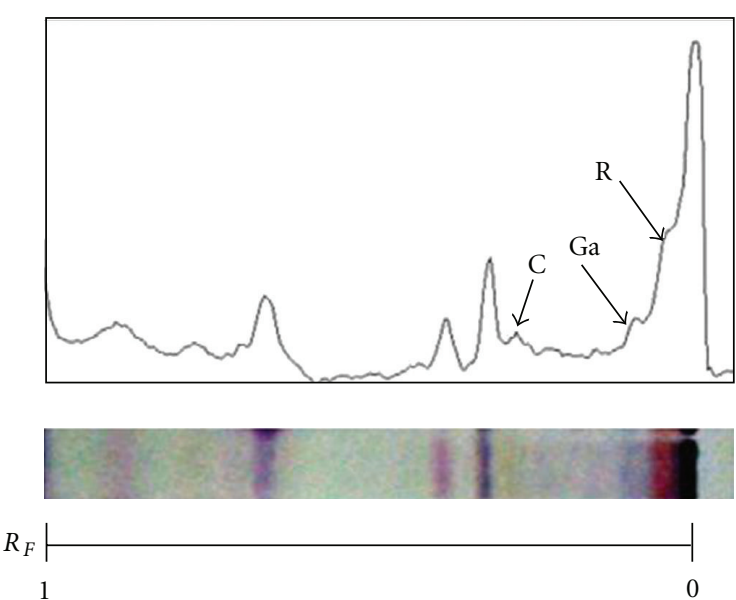

(b)
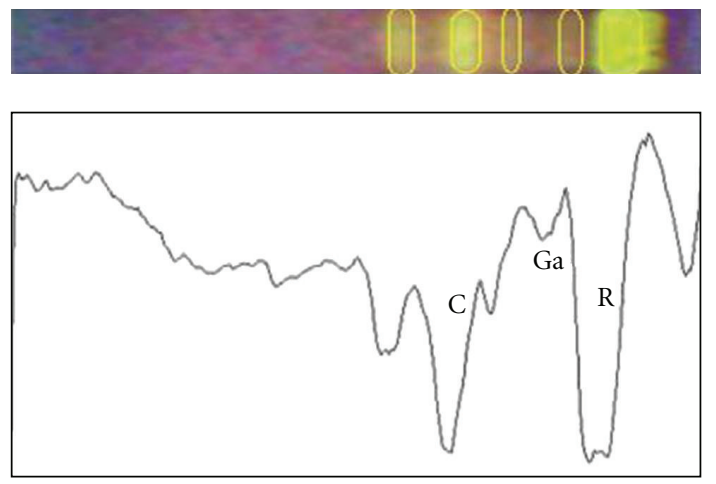

(d)

FIGURE 5: Comparison of chemical and free radical scavenging TLC fingerprints obtained with use of ImageJ program, for the extracts prepared from two Salvia species: (a) S. lavandulaefolia and (b) S. atropatana. Symbols' meaning: C:caffeic acid, Ga: gallic acid, R: rosmarinic acid (originally published in [25]).

the results, that are changing in time, as the one obtained with $\mathrm{DPPH}^{\bullet}$ as derivatizing agent [26]. With the application of the technique, four Salvia species were characterized as a rich source of free radical scavengers, active in vitro, namely, S. officinalis, S. triloba, S. canariensis, and S. lavandulaefolia [25]. The comparison of both chemical and free radical scavenging fingerprints led to a conclusion, that S. triloba can be further investigated as a possible equivalent of the pharmacopoeial S. officinalis. The authors underline also that coupling chemical and biological fingerprint enables more comprehensive investigation of the analyzed samples, as some features barely seen in chemical profile may be more distinct in the biological one.

The analogous procedure has been applied for the quality control of pharmaceutical preparations containing Salvia officinalis extract [27]. Both chemical and biological chromatographic fingerprints of properly processed chromatographic formulations were compared with chromatographic profiles obtained for a botanical reference material (BRM). It was concluded that the proposed technique may be successfully applied for the comprehensive quality control of the finished products containing sage extract.
The idea of binary chromatographic fingerprint construction by means of thin-layer chromatography is not a new one, as it had been already described by Chen et al. [28]. However, the concept of binary chromatographic fingerprints in the aforementioned papers is somewhat different. In case of the paper by Chen et al., the term "binary" relates to fingerprints obtained separately for glycoside and aglycone fractions, which are bounded by a peak that appears in both profiles. In the paper by Cieśla et al., the focus is on joining the biological and chemical chromatographic fingerprints in order to obtain more information needed for species differentiation and identification of bioactive compounds.

Effect directed analysis, aimed at the isolation of compounds characterized with desired activity, has been the primary application for biological detection in TLC. However, as already shown, it can also be used for the purposes of the quality control of different herbal samples. The potential of thin-layer chromatography for performing simple benchtop bioassays has been recently reviewed, and perspectives of its further development were outlined by several authors [29-31]. Apart from the aforementioned application of TLC for assessing free radical activity, it 


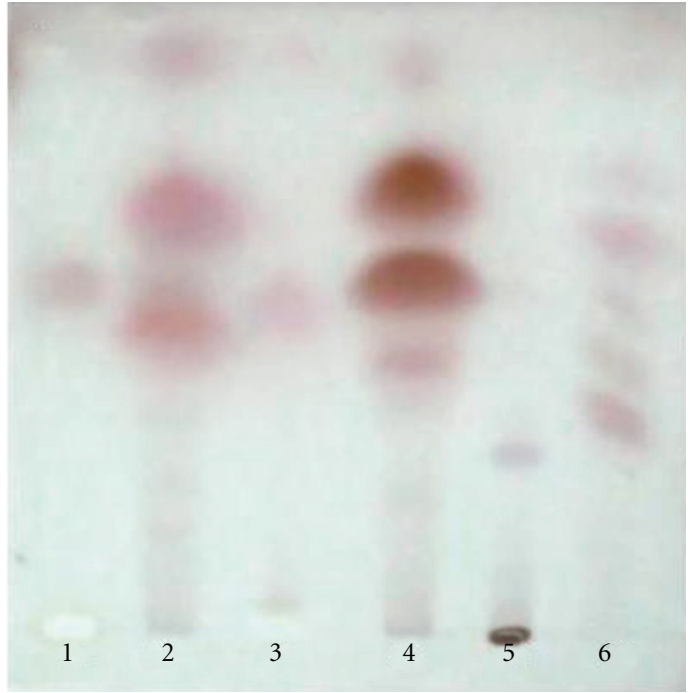

(a)

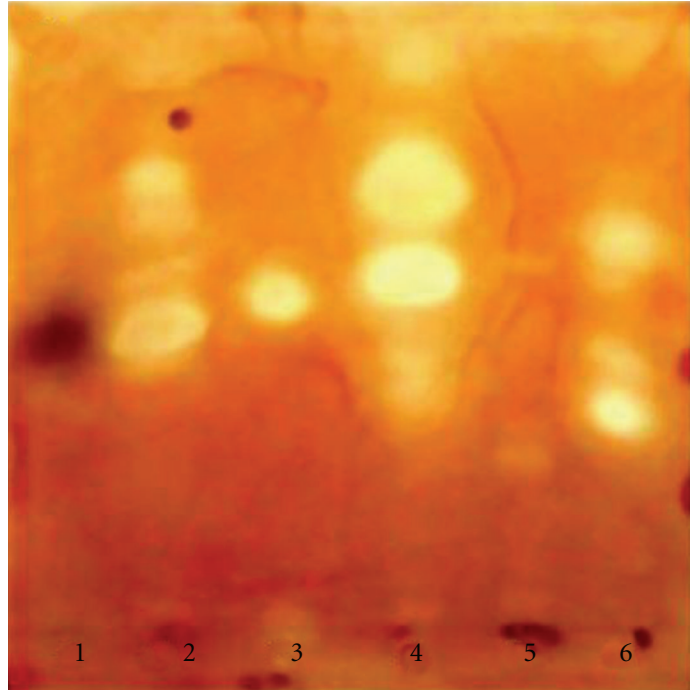

(b)

Figure 6: Comparison of (a) chemical and (b) biological profiles (AchE inhibitory activity) obtained for selected Lamiaceae plant samples. In case of chemical detection the plate was sprayed with $20 \%$ methanolic solution of sulfuric acid; in case of biological profile, the plate was derivatized according to diazotization method [30]. Symbols' meaning: 1: Thymus vulgaris essential oil, 2: Rosmarinus officinalis essential oil, 3: Mentha piperita essential oil, 4: Lavandula officinalis essential oil, 5: Salvia officinalis (ethanolic tincture), and 6: Melissa officinalis essential oil [34].

can also be used to screen the natural samples for the presence of the inhibitors of selected enzymes or to detect compounds with antibacterial or antifungal properties [2931]. Due to the large number of people suffering from neurodegenerative ailments (e.g., Alzheimer's or Parkinson's disease) and limited amount of approved drugs, there is a growing need for finding new medicines. Screening natural samples for the presence of acetylcholinesterase (AChE) inhibitors, by means of simple TLC benchtop bioassays, has recently become very popular among scientists, whose researches are focused on the discovery of new potential drugs to treat Alzheimer's disease. New solutions, for better TLC tests' performance, are still being sought and published [32]. The recent results obtained at the Department of Inorganic Chemistry have shown, it is possible to apply low-temperature TLC for screening volatile samples for the presence of AChE inhibitors [33]. An example of using TLCAChE inhibitory test for selected volatile samples has been shown in Figure 6. Low-temperature TLC bioassays can be a method of choice for effect directed analysis and biological fingerprint construction of volatiles. In case of essential oils, the analytical method of choice is usually GC-MS; however, the use of this technique excludes the possibility of applying effect-directed analysis (detection of direct antioxidants or AChE inhibitors). Thus it may be concluded that TLC biological fingerprinting can be a valuable tool in case of volatile samples, whose content is variable, which may result in changes of its efficacy, when used for medical purposes.

Attention should be paid to rational use of thin-layer chromatographic fingerprint analysis. TLC biofingerprints should only be constructed in case when the analyzed extract or formulation is intended to be used due to its properties screened in the study. For example, there is no need to perform AChE inhibitory tests for samples that will never be used for treating dementia of Alzheimer's type. Recently, the abuse of different $\mathrm{DPPH}^{\bullet}$ (including TLC-DPPH ${ }^{\bullet}$ tests) screening techniques can be observed. In many papers, a direct link between the results of $\mathrm{DPPH}^{\bullet}$ studies and pharmacological activity is claimed, which is not supported by any scientific data. The easiness of TLC-DPPH ${ }^{\bullet}$ test performance can be one of the reasons of its popularity. The most common abuses of simple in vitro tests have been described by Houghton et al. [35]. An important issue, that should also be taken into account in case of biological fingerprints, is false-positive results. They may appear for example, due to interactions of analyzed compounds with the active sites of the used adsorbents. Such interactions have been observed while performing tests aimed at the detection of acetylcholinesterase inhibitors or free radical scavengers in plant extracts $[33,36,37]$.

\section{Perspectives of BFA Development}

As it was already stated, the idea of biofingerprint is a new concept in the analysis of multicomponent herbal samples; therefore, its potential for further development is great. In the future, HPLC-based biofingerprint analysis can be extended to screen herbal samples for the presence of bioactive compounds that can interact with particular receptors. New solutions may also be proposed to overcome the common problems encountered in studying analyteenzyme interactions. Novel detection techniques as well as hyphenations may also be proposed to detect bioactive 
compounds present in plant extracts. Yu et al. describe the potential of coupling BFA with omics technologies in the discovery of bioactive compounds in traditional Chinese medicines [5]. Thus BFA can be regarded as a powerful and still developing tool in drug discovery.

In case of planar chromatography, special modes of chromatogram development (e.g., multidimensional and multimodal separations, two-dimensional TLC) can be combined with chemical and biological detection for the purposes of effect directed analysis (EDA) as well as fingerprint construction. This approach may be beneficial especially in the case of very complex samples, for example, polyherbal formulations. Special modes of chromatogram development have been well described in the literature $[38,39]$ with examples of their application for fingerprint construction [40, 41]. Supervised and unsupervised chemometric analysis of two-dimensional images has also been recently described that turned out to be beneficial for recognizing the differences between closely related plant species [42]. Apart from chemometric pretreatment, a concept of an average fingerprint construction by means of freely available program ImageJ (Wayne Rasband, Natonal Institutes of Health, USA; http://rsbweb.nih.gov/ij/) has also been proposed [43]. This idea has been introduced to overcome the problem of the $R_{\mathrm{F}}$ values shift encountered in TLC. The average fingerprint is constructed with the use of "Calculator" function of the ImageJ program. The detailed description of the procedure can be found elsewhere [43]. Planar chromatography is ideally suited for different hyphenations, and even superhyphenations (hypernations) [44]. Coupling low-temperature TLC with MS detection and gas chromatography has recently been shown as suitable for fingerprint construction of essential oils from different Salvia species $[45,46]$. All the aforementioned solutions may also be further applied in biological fingerprint analysis. the first report that describes the combination of two-dimensional TLC with DPPH ${ }^{\bullet}$ staining, in the analysis of secondary plant metabolites, has already been published [47]. The authors show also an interesting way of data presentation with the use of ImageJ program processing (Figure 7).

Coupling biological detection with fingerprint construction in TLC may in future even outperform some biological fingerprint solutions offered by HPLC.

\section{Conclusions}

Biological detection in liquid chromatography gives an opportunity to comprehensively analyze herbal samples. Apart from greater amount of data, biofingerprints enable screening plant samples for the presence of new active compounds (effect-directed analysis). It is possible to distinguish the bioactive compounds from among the set of chromatographic and spectroscopic signals. HPLC and TLC can be regarded as complementary techniques, as some biofingerprint solutions are easier to be realized by means of HPLC, in other cases TLC may outperform HPLC. For example, affinity chromatography (DNA-target analysis, HSA binding, or liposome-binding chromatography) can

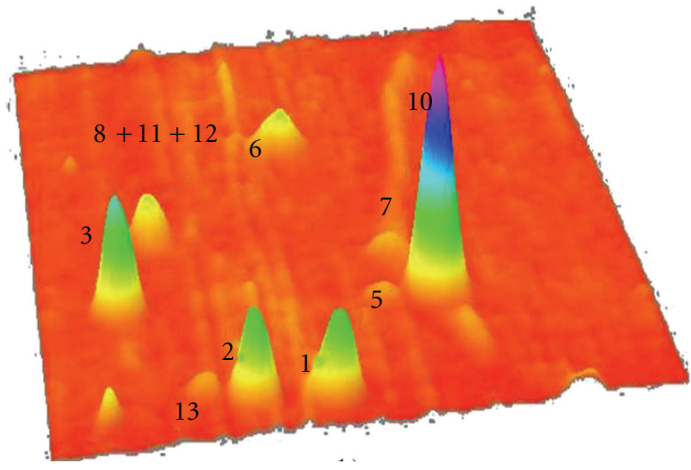

Figure 7: Three dimensional plot obtained for a set of standard compounds separated by means of two-dimensional thin-layer chromatography. For symbols see the original publication [47].

be performed by means of HPLC. Construction of volatile samples' biofingerprints is possible to be realized by means of low-temperature TLC. TLC-based screening techniques, for potential plant-derived enzyme inhibitors still outperform those based on HPLC separations. What is more libraries of active compounds may be created with the use of liquid chromatography biofingerprints. HPLC and TLC can be both used in dereplication step, aimed at avoiding isolation of compounds with proven activity. However, the real potential of biological fingerprinting is yet to be explored.

\section{Acknowledgment}

Financial support from the 7FP EC project "Proficiency" is gratefully acknowledged.

\section{References}

[1] C. Tistaert, B. Dejaegher, and Y. V. Heyden, "Chromatographic separation techniques and data handling methods for herbal fingerprints: a review," Analytica Chimica Acta, vol. 690, no. 2, pp. 148-161, 2011.

[2] WHO, General Guidelines for Methodologies on Research and Evaluation of Traditional Medicine, World Health Organization, Geneva, Switzerland, 2000.

[3] X. H. Fan, Y. Y. Cheng, Z. L. Ye, R. C. Lin, and Z. Z. Qian, "Multiple chromatographic fingerprinting and its application to the quality control of herbal medicines," Analytica Chimica Acta, vol. 555, no. 2, pp. 217-224, 2006.

[4] X. Su, L. Kong, X. Lei, L. Hu, M. Ye, and H. Zou, "Biological fingerprinting analysis of traditional Chinese medicines with targeting ADME/Tox property for screening of bioactive compounds by chromatographic and MS methods," MiniReviews in Medicinal Chemistry, vol. 7, no. 1, pp. 87-98, 2007.

[5] F. Yu, L. Kong, H. Zou, and X. Lei, "Progress on the screening and analysis of bioactive compounds in traditional Chinese medicines by biological fingerprinting analysis," Combinatorial Chemistry and High Throughput Screening, vol. 13, no. 10, pp. 855-868, 2010.

[6] X. Lei, L. Kong, H. Zou, H. Ma, and L. Yang, "Evaluation of the interaction of bioactive compounds in Cortex Pseudolarix and Radix Stephaniae by the microdialysis probe coupled with 
high performance liquid chromatography-mass spectrometry," Journal of Chromatography A, vol. 1216, no. 11, pp. 21792184, 2009.

[7] X. Su, L. Hu, L. Kong, X. Lei, and H. Zou, "Affinity chromatography with immobilized DNA stationary phase for biological fingerprinting analysis of traditional Chinese medicines," Journal of Chromatography A, vol. 1154, no. 1-2, pp. 132-137, 2007.

[8] X. Su, L. Kong, X. Li, X. Chen, M. Guo, and H. Zou, "Screening and analysis of bioactive compounds with biofingerprinting chromatogram analysis of traditional Chinese medicines targeting DNA by microdialysis/HPLC," Journal of Chromatography A, vol. 1076, no. 1-2, pp. 118-126, 2005.

[9] Y. Wang, L. Kong, L. Hu et al., "Biological fingerprinting analysis of the traditional Chinese prescription Longdan Xiegan Decoction by on/off-line comprehensive two-dimensional biochromatography," Journal of Chromatography B, vol. 860, no. 2, pp. 185-194, 2007.

[10] M. Guo, X. Su, L. Kong, X. Li, and H. Zou, "Characterization of interaction property of multicomponents in Chinese Herb with protein by microdialysis combined with HPLC," Analytica Chimica Acta, vol. 556, no. 1, pp. 183-188, 2006.

[11] X. Mao, L. Kong, Q. Luo, X. Li, and H. Zou, "Screening and analysis of permeable compounds in Radix Angelica Sinensis with immobilized liposome chromatography," Journal of Chromatography B, vol. 779, no. 2, pp. 331-339, 2002.

[12] Y. Wang, L. Kong, X. Lei et al., "Comprehensive twodimensional high-performance liquid chromatography system with immobilized liposome chromatography column and reversed-phase column for separation of complex traditional Chinese medicine Longdan Xiegan Decoction," Journal of Chromatography A, vol. 1216, no. 11, pp. 2185-2191, 2009.

[13] J. L. Zhang, M. Cui, Y. He, H. L. Yu, and D. A. Guo, "Chemical fingerprint and metabolic fingerprint analysis of Danshen injection by HPLC-UV and HPLC-MS methods," Journal of Pharmaceutical and Biomedical Analysis, vol. 36, no. 5, pp. 1029-1035, 2005.

[14] H. A. G. Niederländer, T. A. van Beek, A. Bartasiute, and I. I. Koleva, "Antioxidant activity assays on-line with liquid chromatography," Journal of Chromatography A, vol. 1210, no. 2, pp. 121-134, 2008.

[15] M. Musialik, R. Kuzmicz, T. S. Pawlowski, and G. Litwinienko, "Acidity of hydroxyl groups: an overlooked influence on antiradical properties of flavonoids," Journal of Organic Chemistry, vol. 74, no. 7, pp. 2699-2709, 2009.

[16] Y. X. Chang, X. P. Ding, J. Qi et al., "The antioxidant-activityintegrated fingerprint: an advantageous tool for the evaluation of quality of herbal medicines," Journal of Chromatography A, vol. 1208, no. 1-2, pp. 76-82, 2008.

[17] T. A. van Beek, K. K. R. Tetala, I. I. Koleva et al., "Recent developments in the rapid analysis of plants and tracking their bioactive constituents," Phytochemistry Reviews, vol. 8, no. 2, pp. 387-399, 2009.

[18] A. M. van Nederkassel, M. Daszykowski, D. L. Massart, and Y. Vander Heyden, "Prediction of total green tea antioxidant capacity from chromatograms by multivariate modeling," Journal of Chromatography A, vol. 1096, no. 1-2, pp. 177-186, 2005.

[19] M. Daszykowski, Y. Vander Heyden, and B. Walczak, "Robust partial least squares model for prediction of green tea antioxidant capacity from chromatograms," Journal of Chromatography A, vol. 1176, no. 1-2, pp. 12-18, 2007.

[20] M. Dumarey, A. M. van Nederkassel, E. Deconinck, and
Y. Vander Heyden, "Exploration of linear multivariate calibration techniques to predict the total antioxidant capacity of green tea from chromatographic fingerprints," Journal of Chromatography A, vol. 1192, no. 1, pp. 81-88, 2008.

[21] N. Nguyen Hoai, B. Dejaegher, C. Tistaert et al., "Development of HPLC fingerprints for Mallotus species extracts and evaluation of the peaks responsible for their antioxidant activity," Journal of Pharmaceutical and Biomedical Analysis, vol. 50, no. 5, pp. 753-763, 2009.

[22] C. Tistaert, B. Dejaegher, N. Nguyen Hoai et al., "Potential antioxidant compounds in Mallotus species fingerprints. Part I: indication, using linear multivariate calibration techniques," Analytica Chimica Acta, vol. 649, no. 1, pp. 24-32, 2009.

[23] M. Dumarey, I. Smets, and Y. Vander Heyden, "Prediction and interpretation of the antioxidant capacity of green tea from dissimilar chromatographic fingerprints," Journal of Chromatography B, vol. 878, no. 28, pp. 2733-2740, 2010.

[24] E. Reich and A. Schibli, High-Performance Thin-Layer Chromatography for the Analysis of Medicinal Plants, Thieme, New York, NY, USA, 2006.

[25] Ł. Cieśla, D. Staszek, M. Hajnos, T. Kowalska, and M. Waksmundzka-Hajnos, "Development of chromatographic and free radical scavenging activity fingerprints by thin-layer chromatography for selected Salvia species," Phytochemical Analysis, vol. 22, no. 1, pp. 59-65, 2011.

[26] T. Yrjönen, L. Peiwu, J. Summanen, A. Hopia, and H. Vuorela, "Free radical-scavenging activity of phenolics by reversedphase TLC," Journal of the American Oil Chemists' Society, vol. 80, no. 1, pp. 9-14, 2003.

[27] Ł. Cieśla and M. Waksmundzka-Hajnos, "Application of thinlayer chromatography for the quality control and screening the free radical scavenging activity of selected pharmacuetical preparations containing Salvia officinalis L. extract," Acta Poloniae Pharmaceutica-Drug Research, vol. 67, no. 5, pp. 481-485, 2010.

[28] S. B. Chen, H. P. Liu, R. T. Tian et al., "High-performance thin-layer chromatographic fingerprints of isoflavonoids for distinguishing between Radix Puerariae Lobate and Radix Puerariae Thomsonii," Journal of Chromatography A, vol. 1121, no. 1, pp. 114-119, 2006.

[29] Ł. Cieśla, "Thin-layer chromatography with biodetection for the search of new potential drugs to treat neurodegenerative diseases - state of the art and future perspectives," Medicinal Chemistry. In press.

[30] A. Marston, "Thin-layer chromatography with biological detection in phytochemistry," Journal of Chromatography A, vol. 1218, no. 19, pp. 2676-2683, 2011.

[31] I. M. Choma and E. M. Grzelak, "Bioautography detection in thin-layer chromatography," Journal of Chromatography A, vol. 1218, no. 19, pp. 2684-2691, 2011.

[32] Z.-D. Yang, Z.-W. Song, J. Ren, M.-J. Yang, and S. Li, "Improved thin-layer chromatography bioautographic assay for the detection of acetylcholinesterase inhibitors in plants," Phytochemical Analysis, vol. 22, no. 6, pp. 509-515, 2011.

[33] Ł. Cieśla, J. Kryszeń, A. Stochmal, and M. WaksmundzkaHajnos, "TLC-DPPH test revisited," in Proceedings of the 34th Symposium: Chromatographic Methods of Investigating the Organic Compounds, Katowice-Szczyrk, Poland, June 2011.

[34] Ł. Cieśla, J. Kryszeń, and M. Waksmundzka-Hajnos, “unpublished results".

[35] P. J. Houghton, M. J. Howes, C. C. Lee, and G. Steventon, "Uses and abuses of in vitro tests in ethnopharmacology: visualizing an elephant," Journal of Ethnopharmacology, vol. 110, no. 3, pp. 391-400, 2007. 
[36] I. K. Rhee, M. van de Meent, K. Ingkaninan, and R. Verpoorte, "Screening for acetylcholinesterase inhibitors from Amaryllidaceae using silica gel thin-layer chromatography in combination with bioactivity staining," Journal of Chromatography $A$, vol. 915, no. 1-2, pp. 217-223, 2001.

[37] S. Di Giovanni, A. Borloz, A. Urbain et al., "In vitro screening assays to identify natural or synthetic acetylcholinesterase inhibitors: thin layer chromatography versus microplate methods," European Journal of Pharmaceutical Sciences, vol. 33, no. 2, pp. 109-119, 2008.

[38] Ł. Cieśla and M. Waksmundzka-Hajnos, "Two-dimensional thin-layer chromatography in the analysis of secondary plant metabolites," Journal of Chromatography A, vol. 1216, no. 7, pp. 1035-1052, 2009.

[39] Ł. Cieśla and M. Waksmundzka-Hajnos, "Multidimensional and multimodal separations by HPTLC in phytochemistry," in High-Performance Thin-Layer Chromatography (HPTLC), M. M. Srivastava, Ed., Springer, Berlin, Germany, 2011.

[40] Ł. Cieśla, A. Bogucka-Kocka, M. Hajnos, A. Petruczynik, and M. Waksmundzka-Hajnos, "Two-dimensional thin-layer chromatography with adsorbent gradient as a method of chromatographic fingerprinting of furanocoumarins for distinguishing selected varieties and forms of Heracleum spp," Journal of Chromatography A, vol. 1207, no. 1-2, pp. 160-168, 2008.

[41] L. Cieśla, K. Skalicka-Woźniak, M. Hajnos, M. Hawrył, and M. Waksmundzka-Hajnos, "Multidimensional TLC procedure for separation of complex natural mixtures spanning a wide polarity range; Application for fingerprint construction and for investigation of systematic relationships within the Peucedanum genus," Acta Chromatographica, vol. 21, no. 4, pp. 641-657, 2009.

[42] Ł. Komsta, T. Cieśla, A. Bogucka-Kocka, A. Józefczyk, J. Kryszeń, and M. Waksmundzka-Hajnos, "The start-to-end chemometric image processing of 2D thin-layer videoscans," Journal of Chromatography A, vol. 1218, no. 19, pp. 2820-2825, 2011.

[43] L. Cieśla, M. Hajnos, and M. Waksmundzka-Hajnos, "Application of hydrophilic interaction TLC systems for separation of highly polar glycosidic compounds from the flowers of selected Verbascum species," Journal of Planar Chromatography-Modern TLC, vol. 24, no. 4, pp. 295-300, 2011.

[44] G. Morlock and W. Schwack, "Hyphenations in planar chromatography," Journal of Chromatography A, vol. 1217, no. 43, pp. 6600-6609, 2010.

[45] M. Sajewicz, Ł. Wojtal, M. Hajnos, M. Waksmundzka-Hajnos, and T. Kowalska, "Low-temperature TLC-MS of essential oils from five different sage (Salvia) species," Journal of Planar Chromatography-Modern TLC, vol. 23, no. 4, pp. 270-276, 2010.

[46] M. Sajewicz, L. Wojtal, D. Staszek, M. Hajnos, M. Waksmundzka-Hajnos, and T. Kowalska, "Low temperature planar chromatography-densitometry and gas chromatography of essential oils from different sage (Salvia) species," Journal of Liquid Chromatography and Related Technologies, vol. 33, no. 7-8, pp. 936-947, 2010.

[47] M. A. Hawrył and M. Waksmundzka-Hajnos, "Twodimensional thin-layer chromatography of selected Polygonum sp. extracts on polar-bonded stationary phases," Journal of Chromatography A, vol. 1218, no. 19, pp. 2812-2819, 2011. 


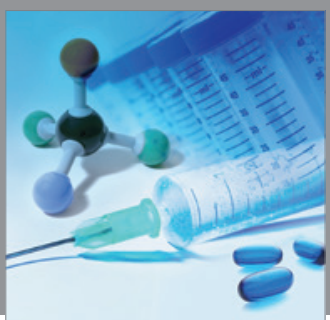

International Journal of

Medicinal Chemistry

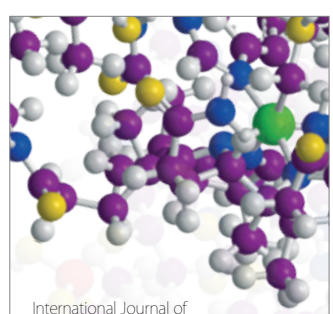

Carbohydrate Chemistry

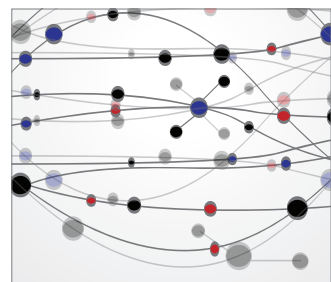

The Scientific World Journal
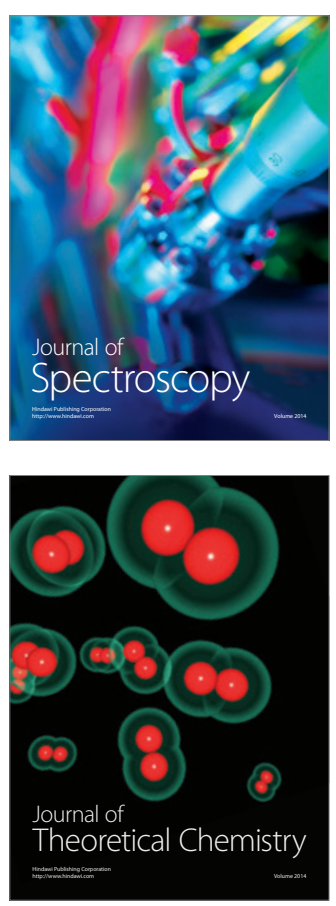
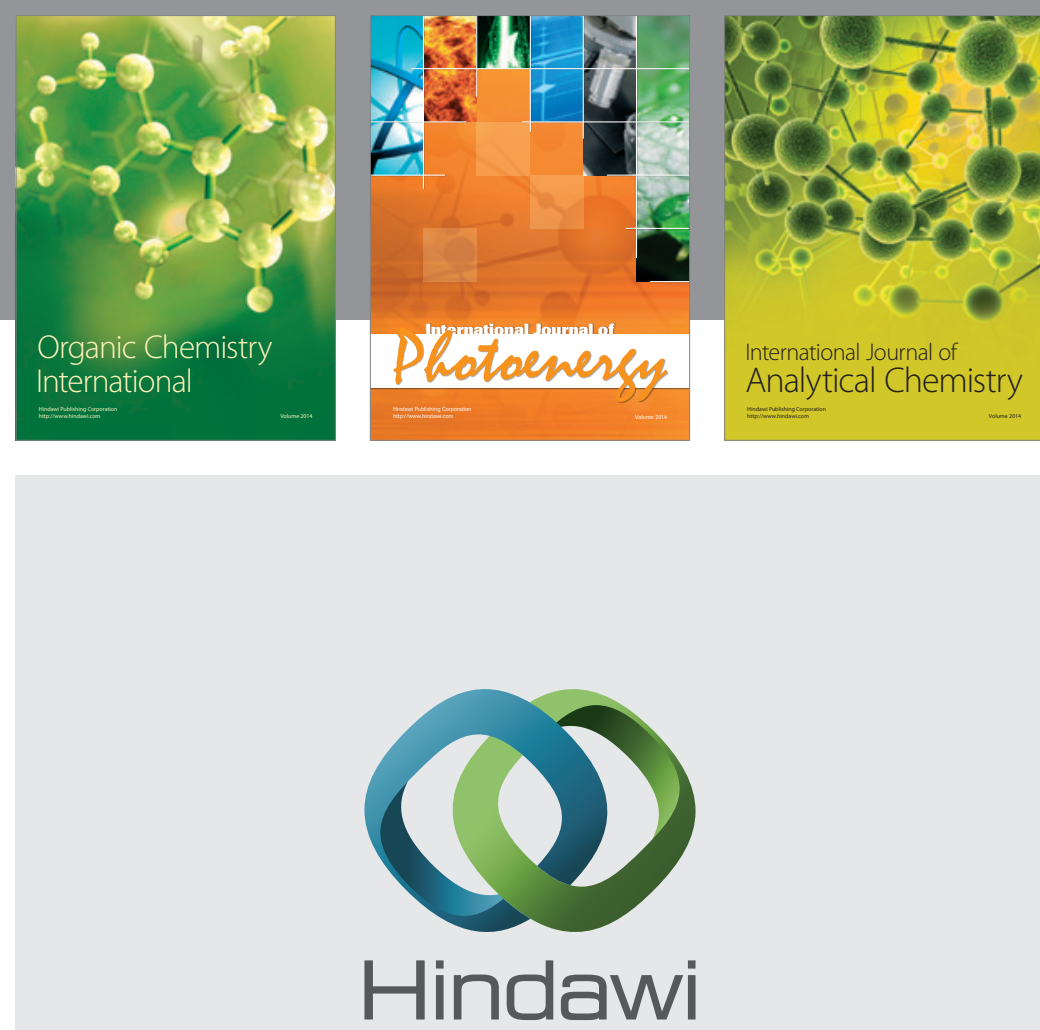

Submit your manuscripts at

http://www.hindawi.com
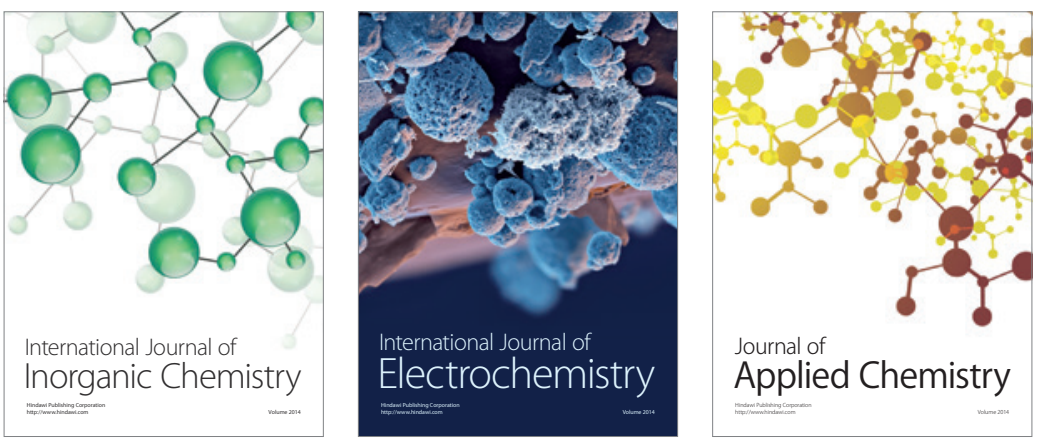

Journal of

Applied Chemistry
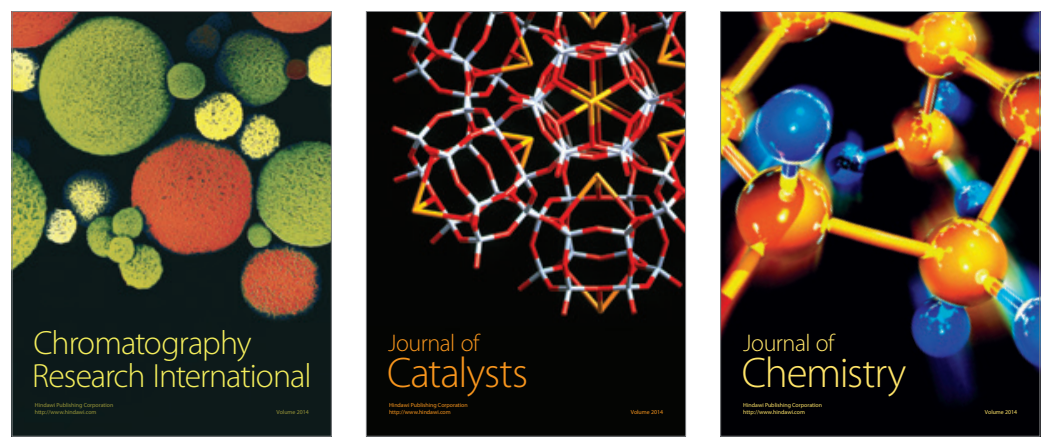
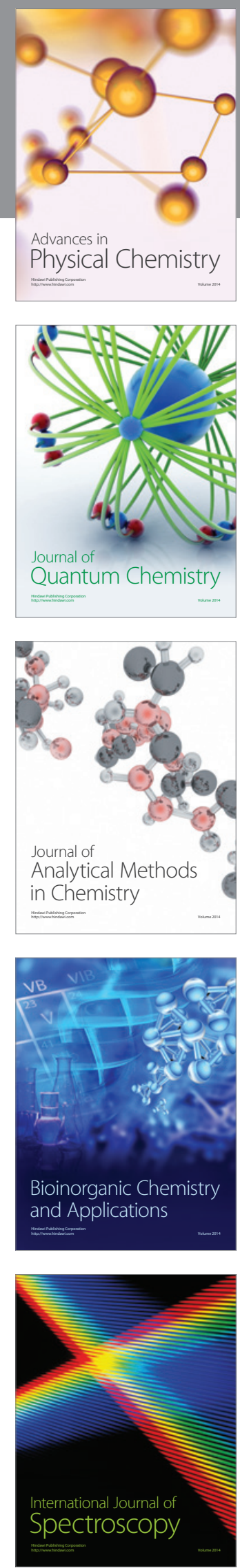